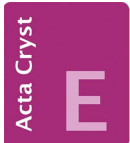

CRYSTALLOGRAPHIC COMMUNICATIONS

ISSN 2056-9890

Received 18 September 2017

Accepted 20 September 2017

Edited by W. T. A. Harrison, University of Aberdeen, Scotland

Keywords: crystal structure; isotypism; sodium arsenate; hydrogen bonding.

CCDC reference: 1575494

Supporting information: this article has supporting information at journals.iucr.org/e

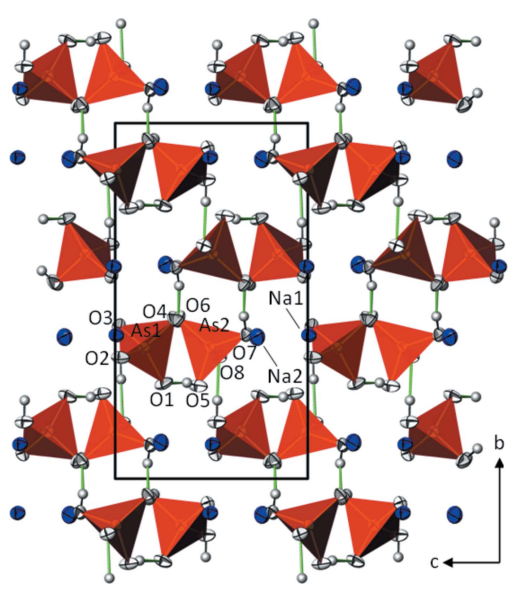

OPEN $\odot$ ACCESS

\section{Crystal structure of sodium dihydrogen arsenate}

\author{
Joseph Ring, ${ }^{a}$ Lorenz Lindenthal, ${ }^{a}$ Matthias Weil ${ }^{b *}$ and Berthold Stöger ${ }^{\mathrm{c}}$
}

${ }^{a}$ TU Wien, Getreidemarkt 9/164-SC, A-1060 Vienna, Austria, ${ }^{\mathbf{b}}$ Institute for Chemical Technologies and Analytics,
Division of Structural Chemistry, TU Wien, Getreidemarkt 9/164-SC, A-1060 Vienna, Austria, and ${ }^{\mathbf{c} X-R a y ~ C e n t r e, ~ T U ~}$
Wien, Getreidemarkt 9, A-1060 Vienna, Austria. ${ }^{*}$ Correspondence e-mail: matthias.weil@tuwien.ac.at

Single crystals of the title compound, $\mathrm{Na}\left(\mathrm{H}_{2} \mathrm{AsO}_{4}\right)$, were obtained by partial neutralization of arsenic acid with sodium hydroxide in aqueous solution. The crystal structure of $\mathrm{Na}\left(\mathrm{H}_{2} \mathrm{AsO}_{4}\right)$ is isotypic with the phosphate analogue and the asymmetric unit consists of two sodium cations and two tetrahedral $\mathrm{H}_{2} \mathrm{AsO}_{4}{ }^{-}$ anions. Each of the sodium cations is surrounded by six $\mathrm{O}$ atoms of five $\mathrm{H}_{2} \mathrm{AsO}_{4}{ }^{-}$groups, defining distorted octahedral coordination spheres. In the extended structure, the sodium cations and dihydrogen arsenate anions are arranged in the form of layers lying parallel to (010). Strong hydrogen bonds [range of $\mathrm{O} \cdots \mathrm{O}$ distances 2.500 (3)-2.643 (3) $\AA$ ] between adjacent $\mathrm{H}_{2} \mathrm{AsO}_{4}{ }^{-}$ anions are observed within and perpendicular to the layers. The isotypic structure of $\mathrm{Na}\left(\mathrm{H}_{2} \mathrm{PO}_{4}\right)$ is comparatively discussed.

\section{Chemical context}

Arsenic acid is triprotic and thus can form various salts, depending on the degree of deprotonation $\left(\mathrm{H}_{2} \mathrm{AsO}_{4}{ }^{-}\right.$, $\mathrm{HAsO}_{4}{ }^{2-}, \mathrm{AsO}_{4}{ }^{3-}$ ), the condensation grade of the anion (mono-, di-, tri-, polyarsenate, etc) and the amount of water incorporated in the crystal. With respect to sodium arsenates, numerous crystal structures have been determined so far, including arsenic in tetrahedral and/or in octahedral coordination by oxygen atoms. Arsenate structures with arsenic exclusively in tetrahedral coordination resemble those of the related phosphates and in some cases show isotypism with them (marked by an asterisk): $\mathrm{Na}_{3.25}\left(\mathrm{AsO}_{4}\right)(\mathrm{OH})_{0.25}\left(\mathrm{H}_{2} \mathrm{O}\right)_{12}$ * (Tillmanns \& Baur, 1971), $\mathrm{Na}_{4}\left(\mathrm{AsO}_{4}\right) \mathrm{OH}$ (zur Loye et al., 2015), $\mathrm{Na}_{2}\left(\mathrm{HAsO}_{4}\right)\left(\mathrm{H}_{2} \mathrm{O}\right)_{7} *$ (Baur \& Khan, 1970; Ferraris et al., 1971), $\mathrm{Na}\left(\mathrm{H}_{2} \mathrm{AsO}_{4}\right)\left(\mathrm{H}_{2} \mathrm{O}\right)$ (Ferraris et al., 1974), $\mathrm{Na}_{3}\left(\mathrm{H}_{2} \mathrm{As}_{3} \mathrm{O}_{10}\right)$ (Driss \& Jouini, 1990), $\mathrm{Na}_{4} \mathrm{As}_{2} \mathrm{O}_{7}$ (Leung \& Calvo, 1973), $\mathrm{Na}\left(\mathrm{AsO}_{3}\right)$ (Liebau, 1956) and $\mathrm{Na}_{5}\left(\mathrm{AsO}_{5}\right)$ (Haas $\&$ Jansen, 2001). Arsenate structures with arsenic in (complete or partial) octahedral coordination include $\mathrm{Na}\left(\mathrm{H}_{2} \mathrm{As}_{3} \mathrm{O}_{9}\right)$ (Driss, Jouini, Durif et al., 1988), $\mathrm{Na}_{3}\left(\mathrm{H}_{5} \mathrm{As}_{4} \mathrm{O}_{14}\right)$ (Driss \& Jouini, 1989), $\mathrm{Na}\left(\mathrm{HAs}_{2} \mathrm{O}_{6}\right)$ (Dung \& Tahar, 1978), $\mathrm{Na}_{2} \mathrm{As}_{4} \mathrm{O}_{11}$ (Driss, Jouini \& Omezzine, 1988) and $\mathrm{Na}_{7} \mathrm{As}_{11} \mathrm{O}_{31}$ (Guesmi et al., 2006). A detailed discussion of the structural principles and crystal chemical characteristics of arsenates with arsenic in octahedral coordination was given some time ago by Schwendtner \& Kolitsch (2007).

Besides the Na:As 1:1 phase $\mathrm{Na}\left(\mathrm{H}_{2} \mathrm{AsO}_{4}\right)\left(\mathrm{H}_{2} \mathrm{O}\right)$ another 1:1 phase, $\mathrm{Na}\left(\mathrm{H}_{2} \mathrm{AsO}_{4}\right)$, has been reported but without an additional water molecule (Fehér \& Morgenstern, 1937). To our surprise, a detailed structural investigation of this salt has not yet been reported. Therefore, we started crystal growth experiments and determined its structure and report here on the results. 


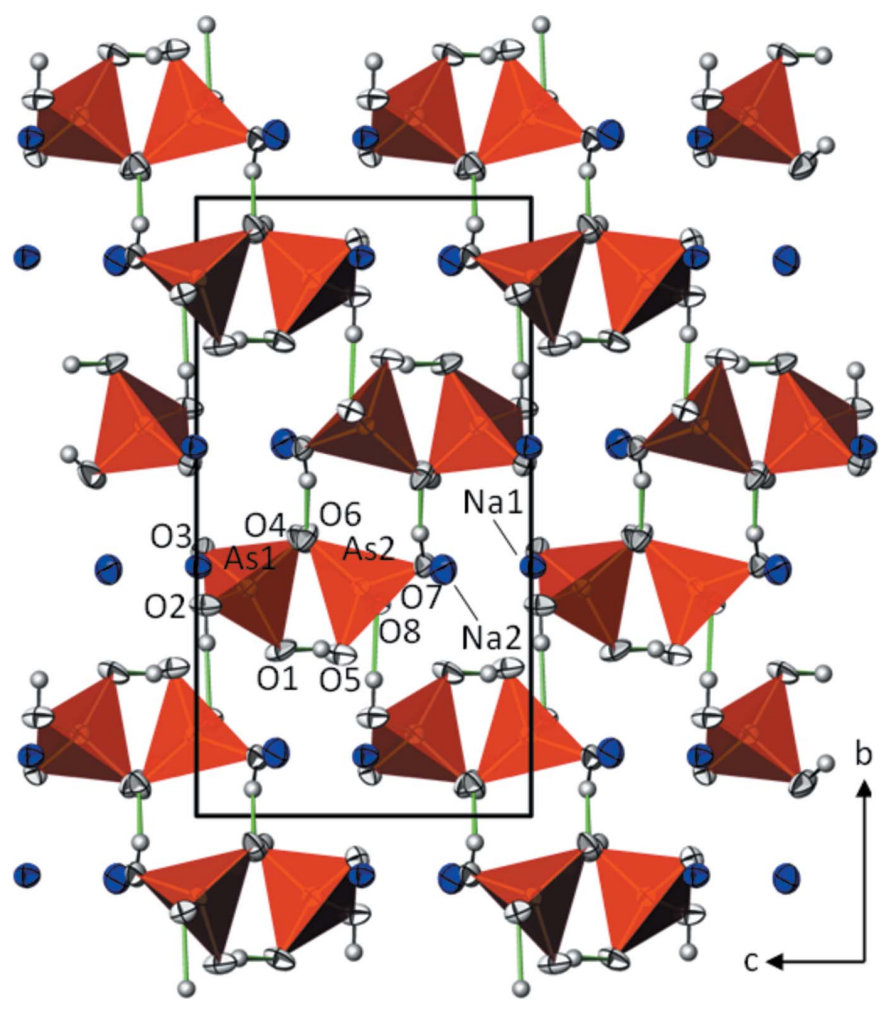

Figure 1

The crystal structure of $\mathrm{Na}\left(\mathrm{H}_{2} \mathrm{AsO}_{4}\right)$ in a projection along [100]. All atoms are depicted with displacement ellipsoids at the $97 \%$ probability level. Dihydrogen arsenate tetrahedra are given in polyhedral representation, $\mathrm{Na}^{+}$cations as single ellipsoids without bonds to surrounding $\mathrm{O}$ atoms. $\mathrm{H} \cdots \mathrm{O}$ hydrogen bonds are illustrated with green lines.

\section{Structural commentary}

The crystal structure of $\mathrm{Na}\left(\mathrm{H}_{2} \mathrm{AsO}_{4}\right)$ is isotypic with that of $\mathrm{Na}\left(\mathrm{H}_{2} \mathrm{PO}_{4}\right)$ (Catti \& Ferraris, 1974). The asymmetric unit of $\mathrm{Na}\left(\mathrm{H}_{2} \mathrm{AsO}_{4}\right)$ comprises two $\mathrm{Na}^{+}$cations and two tetrahedral $\mathrm{AsO}_{2}(\mathrm{OH})_{2}{ }^{-}$groups. The $\mathrm{Na}^{+}{ }^{+}$cation shows a narrow $\mathrm{Na}-\mathrm{O}$ bond-length distribution in the range 2.337 (2) to 2.498 (2) $\AA$ with a distorted octahedron as the corresponding coordination polyhedron. The bond-valence sum (Brown, 2002) for the $\mathrm{Na}^{+}$cation amounts to 1.15 valence units. The surrounding of the $\mathrm{Na}_{2}{ }^{+}$cation is much more distorted, with a bond-length range from 2.338 (2) to 2.769 (3) $\AA$ under consideration of a sixfold coordination (bond-valence sum 0.92 valence units). There is an additional remote oxygen atom at a distance of 3.000 (3) $\AA$ from $\mathrm{Na}_{2}{ }^{+}$. Its contribution of 0.04 valence units to the bond-valence sum might be considered as too low for a significant interaction, and therefore the first coordination sphere of $\mathrm{Na}_{2}^{+}$is discussed as that of a considerably distorted octahedron. The two dihydrogen arsenate groups show the usual differences (Weil, 2000, 2016) between As-O and As$(\mathrm{OH})$ bonds, with two significantly shorter $\mathrm{As}-\mathrm{O}$ bonds [mean $1.659(8) \AA]$ and two longer $\mathrm{As}-(\mathrm{OH})$ bonds [1.723 (12) ̊].

In the crystal structure of $\mathrm{Na}\left(\mathrm{H}_{2} \mathrm{AsO}_{4}\right)$ the $\mathrm{AsO}_{2}(\mathrm{OH})_{2}$ tetrahedra are arranged in layers lying parallel to (010) with the $\mathrm{Na}^{+}$cations approximately on the same level (Fig. 1).
Table 1

Hydrogen-bond geometry $\left(\AA{ }^{\circ}\right)$.

\begin{tabular}{|c|c|c|c|c|}
\hline$D-\mathrm{H} \cdots A$ & $D-\mathrm{H}$ & $\mathrm{H} \cdots A$ & $D \cdots A$ & $D-\mathrm{H} \cdots A$ \\
\hline $\mathrm{O} 2-\mathrm{H} 1 \cdots \mathrm{O} 8^{\mathrm{i}}$ & $0.85(2)$ & $1.75(2)$ & $2.595(3)$ & $175(5)$ \\
\hline $\mathrm{O} 4-\mathrm{H} 2 \cdots \mathrm{O} 8$ & $0.83(2)$ & $1.82(2)$ & $2.643(3)$ & $178(4)$ \\
\hline $\mathrm{O} 5-\mathrm{H} 3 \cdots \mathrm{O} 1^{\mathrm{ii}}$ & $0.85(2)$ & $1.73(2)$ & $2.566(3)$ & $171(5)$ \\
\hline $\mathrm{O} 7-\mathrm{H} 4 \cdots \mathrm{O}^{6 \mathrm{iii}}$ & $0.85(2)$ & $1.66(2)$ & $2.500(3)$ & $169(4)$ \\
\hline
\end{tabular}

Symmetry codes: (i) $x,-y+\frac{1}{2}, z+\frac{1}{2}$; (ii) $x+1, y, z$; (iii) $-x+2,-y+1,-z+1$.

Strong, asymmetric hydrogen bonds $[\mathrm{O} \cdots \mathrm{O}$ distances between 2.500 (3) and 2.643 (3) $\AA$, Table 1] between each of the $\mathrm{OH}$ groups of the two dihydrogen arsenate tetrahedra and $\mathrm{O}$ atoms of adjacent tetrahedra significantly contribute to the crystal packing. These hydrogen bonds are both within a layer and towards adjacent layers (Fig. 1).

The differences between the isotypic arsenate and phosphate structures can mainly be seen in the $X-\mathrm{O}$ bond lengths of the anions ( $X=\mathrm{As}$, mean of $1.69 \AA$; $X=\mathrm{P}$, mean of $1.55 \AA$ ), with $\Delta_{\max }(X-\mathrm{O})$ of $0.15 \AA$ between arsenate and phosphate tetrahedra. The difference with respect to the $\mathrm{Na}-\mathrm{O}$ distances in the two structures is less pronounced, with $\Delta_{\max }(\mathrm{Na}-\mathrm{O})=0.10 \AA$. Relevant bond lengths of the isotypic crystal structures of $\mathrm{Na}\left(\mathrm{H}_{2} \mathrm{AsO}_{4}\right)$ and $\mathrm{Na}\left(\mathrm{H}_{2} \mathrm{PO}_{4}\right)$ (Catti \& Ferraris, 1974) are compiled in Table 2. A more quantitative comparison of the two crystal structures with the help of the COMPSTRU routine (de la Flor et al., 2016) revealed the following values: The degree of lattice distortion $(S)$, i.e. the spontaneous strain (sum of the squared eigenvalues of the strain tensor divided by 3 ), is 0.0159 ; the maximum distance $\left(d_{\text {max }}\right)$, i.e. the maximal displacement between the atomic positions of paired atoms, is $0.1920 \AA$ for atom pair O1; the arithmetic mean $\left(d_{\mathrm{av}}\right)$ of the distances of all atom pairs is

Table 2

Comparison of bond lengths $(\AA)$ in the title compound and the isotypic phosphate analogue (Catti \& Ferraris, 1974).

\begin{tabular}{|c|c|c|}
\hline Bond & $\mathrm{Na}\left(\mathrm{H}_{2} \mathrm{AsO}_{4}\right)$ & $\mathrm{Na}\left(\mathrm{H}_{2} \mathrm{PO}_{4}\right)$ \\
\hline $\mathrm{Na} 1-\mathrm{O} 3^{\mathrm{i}}$ & $2.337(2)$ & $2.355(1)$ \\
\hline $\mathrm{Na} 1-\mathrm{O}^{-\mathrm{ii}}$ & $2.376(2)$ & $2.406(1)$ \\
\hline $\mathrm{Na} 1-\mathrm{O} 3^{\mathrm{iii}}$ & $2.382(2)$ & $2.371(1)$ \\
\hline $\mathrm{Na} 1-\mathrm{O} 7$ & $2.456(2)$ & 2.501 (1) \\
\hline $\mathrm{Na} 1-\mathrm{O} 2^{\mathrm{iv}}$ & $2.459(2)$ & $2.436(1)$ \\
\hline $\mathrm{Na} 1-\mathrm{O}^{\mathrm{iv}}$ & $2.498(2)$ & $2.564(1)$ \\
\hline $\mathrm{Na} 2-\mathrm{O} 8$ & $2.338(2)$ & $2.334(1)$ \\
\hline $\mathrm{Na} 2-\mathrm{O}^{\mathrm{iv}}$ & $2.371(2)$ & 2.369 (1) \\
\hline $\mathrm{Na} 2-\mathrm{O} 1^{\mathrm{ii}}$ & $2.419(2)$ & $2.433(1)$ \\
\hline $\mathrm{Na} 2-\mathrm{O}^{\mathrm{i}}$ & $2.586(2)$ & $2.601(1)$ \\
\hline $\mathrm{Na} 2-\mathrm{O} 2^{\mathrm{iv}}$ & $2.703(3)$ & $2.600(1)$ \\
\hline $\mathrm{Na} 2-\mathrm{O} 4^{\mathrm{i}}$ & 2.769 (3) & $2.730(1)$ \\
\hline $\mathrm{Na} 2-\mathrm{O}_{7}^{\mathrm{v}}$ & $3.000(3)$ & $2.930(1)$ \\
\hline $\mathrm{As} / \mathrm{P} 1-\mathrm{O} 3$ & 1.6484 (19) & 1.499 (1) \\
\hline $\mathrm{As} / \mathrm{P} 1-\mathrm{O} 1$ & $1.657(2)$ & $1.508(1)$ \\
\hline $\mathrm{As} / \mathrm{P} 1-\mathrm{O} 4$ & $1.730(2)$ & $1.592(1)$ \\
\hline $\mathrm{As} / \mathrm{P} 1-\mathrm{O} 2$ & $1.736(2)$ & 1.597 (1) \\
\hline $\mathrm{As} / \mathrm{P} 2-\mathrm{O} 6$ & $1.663(2)$ & $1.523(1)$ \\
\hline $\mathrm{As} / \mathrm{P} 2-\mathrm{O} 8$ & $1.668(2)$ & 1.519 (1) \\
\hline $\mathrm{As} / \mathrm{P} 2-\mathrm{O} 7$ & $1.711(2)$ & $1.562(1)$ \\
\hline $\mathrm{As} / \mathrm{P} 2-\mathrm{O} 5$ & $1.713(2)$ & $1.572(1)$ \\
\hline
\end{tabular}

Symmetry codes (i) $-x+1,-y+1,-z+1$; (ii) $x,-y+\frac{1}{2}, z-\frac{1}{2}$; (iii) $x+1, y, z-1$; (iv) $x, y$, $z-1$; (v) $x-1, y, z$. 
$0.1108 \AA$; the measure of similarity $(\Delta)$ (Bergerhoff et al., 1999) is a function of the differences in atomic positions (weighted by the multiplicities of the sites) and the ratios of the corresponding lattice parameters of the structures and amounts to 0.049 .

\section{Synthesis and crystallization}

The title compound was prepared following a procedure by Fehér \& Morgenstern (1937). An arsenic acid solution (ca $65 \%_{\mathrm{wt}}$ ) was partly neutralized with diluted $\mathrm{NaOH}$ solution using methyl red as indicator. The resulting solution was concentrated by heating. Standing of the solution overnight on a warm plate $(\mathrm{ca} 313 \mathrm{~K})$ afforded colourless crystals with a lath-like form and maximal edge lengths of $0.5 \mathrm{~mm}$.

\section{Refinement}

Crystal data, data collection and structure refinement details are summarized in Table 3. Starting coordinates and labelling of atoms were taken from the isotypic $\mathrm{Na}\left(\mathrm{H}_{2} \mathrm{PO}_{4}\right)$ structure (Catti \& Ferraris, 1974). Hydrogen atoms were clearly discernible from difference maps and were refined with distance restraints $d(\mathrm{O}-\mathrm{H})=0.85(1) \AA$.

\section{Acknowledgements}

The X-ray centre of TU Wien is acknowledged for financial support of this study.

\section{References}

Baur, W. H. \& Khan, A. A. (1970). Acta Cryst. B26, 1584-1596.

Bergerhoff, G., Berndt, M., Brandenburg, K. \& Degen, T. (1999). Acta Cryst. B55, 147-156.

Brown, I. D. (2002). The Chemical Bond in Inorganic Chemistry: The Bond Valence Model. Oxford University Press.

Bruker (2016). APEX3 and SAINT. Bruker AXS Inc., Madison, Wisconsin, USA.

Catti, M. \& Ferraris, G. (1974). Acta Cryst. B30, 1-6.

Dowty, E. (2006). ATOMS. Shape Software, Kingsport, Tennessee, USA.

Driss, A. \& Jouini, T. (1989). J. Solid State Chem. 78, 130-135.

Driss, A. \& Jouini, T. (1990). Acta Cryst. C46, 1185-1188.

Driss, A., Jouini, T., Durif, A. \& Averbuch-Pouchot, M.-T. (1988). Acta Cryst. C44, 1507-1510.

Driss, A., Jouini, T. \& Omezzine, M. (1988). Acta Cryst. C44, 788-791. Dung, N.-H. \& Tahar, J. (1978). Acta Cryst. B34, 3727-3729.

Fehér, F. \& Morgenstern, G. (1937). Z. Anorg. Allg. Chem. 232, 169178.

Ferraris, G., Jones, D. W. \& Sowden, J. M. (1974). Atti R. Accad. Sci. Torino, Cl. Sci. Fis., Mat. Nat. 108, 507-527.
Table 3

Experimental details.

\begin{tabular}{|c|c|}
\hline \multicolumn{2}{|l|}{ Crystal data } \\
\hline Chemical formula & $\mathrm{Na}\left(\mathrm{H}_{2} \mathrm{AsO}_{4}\right)$ \\
\hline$M_{\mathrm{r}}$ & 163.93 \\
\hline Crystal system, space group & Monoclinic, $P 2_{1} / c$ \\
\hline Temperature (K) & 100 \\
\hline$a, b, c(\AA)$ & $7.0528(14), 13.798(3), 7.4792(15)$ \\
\hline$\beta\left(^{\circ}\right)$ & $93.02(3)$ \\
\hline$V\left(\AA^{6}\right)$ & $726.8(3)$ \\
\hline$Z$ & 8 \\
\hline Radiation type & Мо $K \alpha$ \\
\hline$\mu\left(\mathrm{mm}^{-1}\right)$ & 9.32 \\
\hline Crystal size $(\mathrm{mm})$ & $0.12 \times 0.08 \times 0.01$ \\
\hline \multicolumn{2}{|l|}{ Data collection } \\
\hline Diffractometer & Bruker APEXII CCD \\
\hline Absorption correction & $\begin{array}{l}\text { Multi-scan (SADABS; Krause et } \\
\quad \text { al., 2015) }\end{array}$ \\
\hline$T_{\min }, T_{\max }$ & $0.534,0.746$ \\
\hline $\begin{array}{l}\text { No. of measured, independent and } \\
\text { observed }[I>2 \sigma(I)] \text { reflections }\end{array}$ & $11092,2651,1890$ \\
\hline$R_{\text {int }}$ & 0.052 \\
\hline$(\sin \theta / \lambda)_{\max }\left(\AA^{-1}\right)$ & 0.758 \\
\hline \multicolumn{2}{|l|}{ Refinement } \\
\hline$R\left[F^{2}>2 \sigma\left(F^{2}\right)\right], w R\left(F^{2}\right), S$ & $0.030,0.059,1.04$ \\
\hline No. of reflections & 2651 \\
\hline No. of parameters & 125 \\
\hline No. of restraints & 4 \\
\hline $\mathrm{H}$-atom treatment & $\begin{array}{l}\mathrm{H} \text { atoms treated by a mixture of } \\
\text { independent and constrained } \\
\text { refinement }\end{array}$ \\
\hline$\Delta \rho_{\max }, \Delta \rho_{\min }\left(\mathrm{e} \AA^{-3}\right)$ & $0.87,-0.86$ \\
\hline
\end{tabular}

Computer programs: APEX3 and SAINT (Bruker, 2016), SHELXL2016 (Sheldrick, 2015), ATOMS (Dowty, 2006) and publCIF (Westrip, 2010).

Ferraris, G., Jones, D. W. \& Yerkess, J. (1971). Acta Cryst. B27, 354359.

Flor, G. de la, Orobengoa, D., Tasci, E., Perez-Mato, J. M. \& Aroyo, M. I. (2016). J. Appl. Cryst. 49, 653-664.

Guesmi, A., Nespolo, M. \& Driss, A. (2006). J. Solid State Chem. 179, 2466-2471.

Haas, H. \& Jansen, M. (2001). Z. Anorg. Allg. Chem. 627, 10131016.

Krause, L., Herbst-Irmer, R., Sheldrick, G. M. \& Stalke, D. (2015). J. Appl. Cryst. 48, 3-10.

Leung, K. Y. \& Calvo, C. (1973). Can. J. Chem. 51, 2082-2088.

Liebau, F. (1956). Acta Cryst. 9, 811-817.

Loye, K. D. zur, Latshaw, A. M., Smith, M. D., Chance, W. M. \& zur Loye, H. C. (2015). J. Chem. Crystallogr. 45, 20-25.

Schwendtner, K. \& Kolitsch, U. (2007). Acta Cryst. B63, 205-215.

Sheldrick, G. M. (2015). Acta Cryst. C71, 3-8.

Tillmanns, E. \& Baur, W. H. (1971). Acta Cryst. B27, 2124-2132.

Weil, M. (2000). Z. Naturforsch. Teil B, 55, 699-706.

Weil, M. (2016). Cryst. Growth Des. 16, 908-921.

Westrip, S. P. (2010). J. Appl. Cryst. 43, 920-925. 


\section{supporting information}

Acta Cryst. (2017). E73, 1520-1522 [https://doi.org/10.1107/S2056989017013470]

\section{Crystal structure of sodium dihydrogen arsenate}

\section{Joseph Ring, Lorenz Lindenthal, Matthias Weil and Berthold Stöger}

Computing details

Data collection: APEX3 (Bruker, 2016); cell refinement: SAINT (Bruker, 2016); data reduction: SAINT (Bruker, 2016); program(s) used to solve structure: coordinates taken from isotypic structure; program(s) used to refine structure: SHELXL2016 (Sheldrick, 2015); molecular graphics: ATOMS (Dowty, 2006); software used to prepare material for publication: publCIF (Westrip, 2010).

Sodium dihydrogen arsenate

Crystal data

$\mathrm{Na}\left(\mathrm{H}_{2} \mathrm{AsO}_{4}\right)$

$M_{r}=163.93$

Monoclinic, $P 22_{1} / c$

$a=7.0528(14) \AA$

$b=13.798(3) \AA$

$c=7.4792(15) \AA$

$\beta=93.02(3)^{\circ}$

$V=726.8(3) \AA^{3}$

$Z=8$

Data collection

Bruker APEXII CCD

diffractometer

$\omega$ and $\varphi$ scans

Absorption correction: multi-scan

(SADABS; Krause et al., 2015)

$T_{\min }=0.534, T_{\max }=0.746$

11092 measured reflections

\section{Refinement}

Refinement on $F^{2}$

Least-squares matrix: full

$R\left[F^{2}>2 \sigma\left(F^{2}\right)\right]=0.030$

$w R\left(F^{2}\right)=0.059$

$S=1.04$

2651 reflections

125 parameters

4 restraints
$F(000)=624$

$D_{\mathrm{x}}=2.996 \mathrm{Mg} \mathrm{m}^{-3}$

Mo $K \alpha$ radiation, $\lambda=0.71073 \AA$

Cell parameters from 2433 reflections

$\theta=3.1-31.8^{\circ}$

$\mu=9.32 \mathrm{~mm}^{-1}$

$T=100 \mathrm{~K}$

Lath, colourless

$0.12 \times 0.08 \times 0.01 \mathrm{~mm}$

2651 independent reflections 1890 reflections with $I>2 \sigma(I)$

$R_{\text {int }}=0.052$

$\theta_{\text {max }}=32.6^{\circ}, \theta_{\min }=2.9^{\circ}$

$h=-10 \rightarrow 10$

$k=-20 \rightarrow 20$

$l=-11 \rightarrow 11$

Primary atom site location: isomorphous structure methods

Hydrogen site location: difference Fourier map

$\mathrm{H}$ atoms treated by a mixture of independent and constrained refinement

$w=1 /\left[\sigma^{2}\left(F_{\mathrm{o}}^{2}\right)+(0.020 P)^{2}+0.0156 P\right]$

where $P=\left(F_{\mathrm{o}}{ }^{2}+2 F_{\mathrm{c}}{ }^{2}\right) / 3$

$(\Delta / \sigma)_{\max }=0.001$

$\Delta \rho_{\max }=0.87$ e $\AA^{-3}$

$\Delta \rho_{\min }=-0.86$ e $\AA^{-3}$ 


\section{Special details}

Geometry. All esds (except the esd in the dihedral angle between two 1.s. planes) are estimated using the full covariance matrix. The cell esds are taken into account individually in the estimation of esds in distances, angles and torsion angles; correlations between esds in cell parameters are only used when they are defined by crystal symmetry. An approximate (isotropic) treatment of cell esds is used for estimating esds involving l.s. planes.

Fractional atomic coordinates and isotropic or equivalent isotropic displacement parameters $\left(\AA^{2}\right)$

\begin{tabular}{lllll}
\hline & $x$ & $y$ & $z$ & $U_{\text {iso }} * U_{\text {eq }}$ \\
\hline As1 & $0.32467(4)$ & $0.36827(2)$ & $0.84669(4)$ & $0.00611(7)$ \\
As2 & $0.82170(4)$ & $0.37010(2)$ & $0.50756(4)$ & $0.00595(7)$ \\
Na1 & $0.85834(16)$ & $0.40338(8)$ & $-0.00598(15)$ & $0.0091(2)$ \\
Na2 & $0.34819(17)$ & $0.39825(9)$ & $0.26282(16)$ & $0.0134(3)$ \\
O1 & $0.2417(3)$ & $0.26833(14)$ & $0.7478(3)$ & $0.0108(4)$ \\
O2 & $0.5315(3)$ & $0.34069(15)$ & $0.9726(3)$ & $0.0099(4)$ \\
O3 & $0.1910(3)$ & $0.42983(14)$ & $0.9809(3)$ & $0.0078(4)$ \\
O4 & $0.4015(3)$ & $0.44833(15)$ & $0.6878(3)$ & $0.0124(4)$ \\
O5 & $0.9202(3)$ & $0.26110(14)$ & $0.5709(3)$ & $0.0113(4)$ \\
O6 & $0.8540(3)$ & $0.45079(14)$ & $0.6715(3)$ & $0.0098(4)$ \\
O7 & $0.9303(3)$ & $0.40531(15)$ & $0.3188(3)$ & $0.0093(4)$ \\
O8 & $0.5931(3)$ & $0.34459(14)$ & $0.4613(3)$ & $0.0082(4)$ \\
H1 & $0.545(7)$ & $0.2797(14)$ & $0.970(6)$ & $0.055(15)^{*}$ \\
H2 & $0.462(5)$ & $0.415(3)$ & $0.619(5)$ & $0.038(13)^{*}$ \\
H3 & $1.026(4)$ & $0.270(3)$ & $0.627(6)$ & $0.071(18)^{*}$ \\
H4 & $0.995(5)$ & $0.457(2)$ & $0.332(6)$ & $0.045(13)^{*}$ \\
& & & & \\
\hline
\end{tabular}

Atomic displacement parameters $\left(\AA^{2}\right)$

\begin{tabular}{lllllll}
\hline & $U^{11}$ & $U^{22}$ & $U^{33}$ & $U^{12}$ & $U^{13}$ & $U^{23}$ \\
\hline As1 & $0.00551(13)$ & $0.00631(14)$ & $0.00645(13)$ & $0.00067(11)$ & $-0.00029(10)$ & $-0.00017(11)$ \\
As2 & $0.00609(13)$ & $0.00599(14)$ & $0.00564(13)$ & $-0.00119(11)$ & $-0.00081(10)$ & $0.00025(11)$ \\
Na1 & $0.0087(6)$ & $0.0086(5)$ & $0.0099(6)$ & $0.0004(4)$ & $-0.0002(4)$ & $0.0003(4)$ \\
Na2 & $0.0161(6)$ & $0.0132(6)$ & $0.0106(6)$ & $0.0027(5)$ & $-0.0038(5)$ & $-0.0001(5)$ \\
O1 & $0.0101(10)$ & $0.0078(10)$ & $0.0141(11)$ & $-0.0005(8)$ & $-0.0031(8)$ & $-0.0037(8)$ \\
O2 & $0.0077(10)$ & $0.0068(9)$ & $0.0147(11)$ & $0.0005(8)$ & $-0.0044(8)$ & $0.0006(8)$ \\
O3 & $0.0062(10)$ & $0.0094(9)$ & $0.0078(10)$ & $0.0016(7)$ & $0.0007(7)$ & $-0.0011(7)$ \\
O4 & $0.0150(11)$ & $0.0112(10)$ & $0.0114(11)$ & $0.0040(9)$ & $0.0059(9)$ & $0.0033(8)$ \\
O5 & $0.0105(10)$ & $0.0064(10)$ & $0.0163(11)$ & $-0.0006(8)$ & $-0.0054(9)$ & $0.0012(8)$ \\
O6 & $0.0101(10)$ & $0.0110(10)$ & $0.0083(10)$ & $-0.0033(8)$ & $0.0012(8)$ & $-0.0034(8)$ \\
O7 & $0.0122(10)$ & $0.0091(9)$ & $0.0070(9)$ & $-0.0034(9)$ & $0.0038(7)$ & $-0.0008(8)$ \\
O8 & $0.0058(9)$ & $0.0078(9)$ & $0.0108(10)$ & $-0.0011(7)$ & $-0.0013(7)$ & $0.0011(8)$ \\
& & & & & & \\
\hline
\end{tabular}

Geometric parameters $\left(A,{ }^{\circ}\right)$

\begin{tabular}{llll}
\hline As1-O3 & $1.6484(19)$ & $\mathrm{Na} 1-\mathrm{O} 6^{\mathrm{iv}}$ & $2.498(2)$ \\
As1-O1 & $1.657(2)$ & $\mathrm{Na} 2-\mathrm{O} 8$ & $2.338(2)$ \\
As1-O4 & $1.730(2)$ & $\mathrm{Na} 2-\mathrm{O} 3^{\mathrm{iv}}$ & $2.371(2)$ \\
As1-O2 & $1.736(2)$ & $\mathrm{Na} 2-\mathrm{O} 1^{\mathrm{ii}}$ & $2.419(2)$
\end{tabular}




\begin{tabular}{|c|c|c|c|}
\hline As $2-06$ & $1.663(2)$ & $\mathrm{Na} 2-\mathrm{O}^{\mathrm{i}}$ & $2.586(2)$ \\
\hline As $2-\mathrm{O} 8$ & $1.668(2)$ & $\mathrm{Na} 2-\mathrm{O} 2^{\mathrm{iv}}$ & $2.703(3)$ \\
\hline As2-O7 & $1.711(2)$ & $\mathrm{Na} 2-\mathrm{O} 4^{\mathrm{i}}$ & $2.769(3)$ \\
\hline As $2-\mathrm{O} 5$ & $1.713(2)$ & $\mathrm{Na} 2-\mathrm{O}^{\mathrm{v}}$ & $3.000(3)$ \\
\hline $\mathrm{Na} 1-\mathrm{O} 3^{\mathrm{i}}$ & $2.337(2)$ & $\mathrm{O} 2-\mathrm{H} 1$ & $0.847(19)$ \\
\hline $\mathrm{Na} 1-\mathrm{O} 5^{\mathrm{ii}}$ & $2.376(2)$ & $\mathrm{O} 4-\mathrm{H} 2$ & $0.828(18)$ \\
\hline $\mathrm{Na} 1-\mathrm{O} 3^{3 i i}$ & $2.382(2)$ & $\mathrm{O} 5-\mathrm{H} 3$ & $0.847(19)$ \\
\hline $\mathrm{Na} 1-\mathrm{O} 7$ & $2.456(2)$ & $\mathrm{O} 7-\mathrm{H} 4$ & 0.849 (19) \\
\hline $\mathrm{Na} 1-\mathrm{O} 2^{\mathrm{iv}}$ & $2.459(2)$ & & \\
\hline $\mathrm{O} 3-\mathrm{As} 1-\mathrm{O} 1$ & $120.05(10)$ & $\mathrm{O} 1^{\mathrm{ii}}-\mathrm{Na} 2-\mathrm{O} 4^{\mathrm{i}}$ & $157.97(8)$ \\
\hline $\mathrm{O} 3-\mathrm{As} 1-\mathrm{O} 4$ & $107.40(10)$ & $\mathrm{O}^{2}-\mathrm{Na} 2-\mathrm{O}^{\mathrm{i}}$ & $73.32(7)$ \\
\hline $\mathrm{O} 1-\mathrm{As} 1-\mathrm{O} 4$ & $109.92(11)$ & $\mathrm{O} 2^{\mathrm{iv}}-\mathrm{Na} 2-\mathrm{O} 4^{\mathrm{i}}$ & $90.18(7)$ \\
\hline $\mathrm{O} 3-\mathrm{As} 1-\mathrm{O} 2$ & $105.89(10)$ & $\mathrm{O} 8-\mathrm{Na} 2-\mathrm{O} 7^{\mathrm{v}}$ & $128.32(8)$ \\
\hline $\mathrm{O} 1-\mathrm{As} 1-\mathrm{O} 2$ & $109.06(10)$ & $\mathrm{O} 3^{\mathrm{iv}}-\mathrm{Na} 2-\mathrm{O} 7^{\mathrm{v}}$ & $72.63(7)$ \\
\hline $\mathrm{O} 4-\mathrm{As} 1-\mathrm{O} 2$ & $103.17(10)$ & 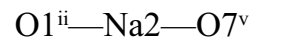 & $74.48(7)$ \\
\hline $\mathrm{O} 6-\mathrm{As} 2-\mathrm{O} 8$ & $112.80(10)$ & $\mathrm{O} 6^{\mathrm{i}}-\mathrm{Na} 2-\mathrm{O} 7^{\mathrm{v}}$ & $52.53(6)$ \\
\hline $\mathrm{O} 6-\mathrm{As} 2-\mathrm{O} 7$ & $111.60(10)$ & $\mathrm{O} 2^{\mathrm{iv}}-\mathrm{Na} 2-\mathrm{O} 7^{\mathrm{v}}$ & $129.53(8)$ \\
\hline $\mathrm{O} 8-\mathrm{As} 2-\mathrm{O} 7$ & $111.00(10)$ & $\mathrm{O} 4-\mathrm{Na} 2-\mathrm{O}^{\mathrm{v}}$ & $125.50(7)$ \\
\hline $\mathrm{O} 6-\mathrm{As} 2-\mathrm{O} 5$ & $110.24(10)$ & As $1-\mathrm{O} 1-\mathrm{Na} 2^{\mathrm{vi}}$ & $131.96(12)$ \\
\hline $\mathrm{O} 8-\mathrm{As} 2-\mathrm{O} 5$ & $104.21(10)$ & As1-O2-Na $1^{\text {vii }}$ & $135.72(11)$ \\
\hline $\mathrm{O} 7-\mathrm{As} 2-\mathrm{O} 5$ & $106.56(10)$ & As1-O2- & $86.99(8)$ \\
\hline $\mathrm{O} 3^{\mathrm{i}}-\mathrm{Na} 1-\mathrm{O} 5^{\mathrm{ii}}$ & $161.36(9)$ & $\mathrm{Na} 1^{\mathrm{vii}}-\mathrm{O} 2-\mathrm{Na} 2^{\mathrm{vii}}$ & $109.31(9)$ \\
\hline 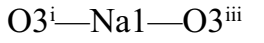 & $90.20(7)$ & $\mathrm{As} 1-\mathrm{O} 2-\mathrm{H} 1$ & $107(3)$ \\
\hline 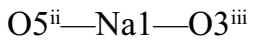 & $89.30(8)$ & $\mathrm{Na}^{\mathrm{vii}}-\mathrm{O} 2-\mathrm{H} 1$ & $104(3)$ \\
\hline $\mathrm{O} 3^{\mathrm{i}}-\mathrm{Na} 1-\mathrm{O} 7$ & $86.19(8)$ & $\mathrm{Na} 2^{\mathrm{vii}}-\mathrm{O} 2-\mathrm{H} 1$ & $111(3)$ \\
\hline $\mathrm{O}^{\mathrm{ii}}-\mathrm{Na} 1-\mathrm{O} 7$ & $75.23(8)$ & $\mathrm{As} 1-\mathrm{O} 3-\mathrm{Na} 1^{\mathrm{i}}$ & $130.46(11)$ \\
\hline  & $83.49(8)$ & As1-O3-Na2 $2^{\text {vii }}$ & $101.02(9)$ \\
\hline $\mathrm{O}^{\mathrm{i}}-\mathrm{Na} 1-\mathrm{O} 2^{\mathrm{iv}}$ & $102.04(8)$ & $\mathrm{Na} 1^{\mathrm{i}}-\mathrm{O} 3-\mathrm{Na} 2^{\mathrm{vii}}$ & $100.03(8)$ \\
\hline $\mathrm{O} 5^{\mathrm{ii}-}-\mathrm{Na} 1-\mathrm{O} 2^{\mathrm{iv}}$ & $80.75(8)$ & $\mathrm{As} 1-\mathrm{O} 3-\mathrm{Na} 1^{\text {viii }}$ & $122.85(11)$ \\
\hline  & $166.71(9)$ & $\mathrm{Na} 1^{\mathrm{i}}-\mathrm{O} 3-\mathrm{Na} 1^{\text {viii }}$ & $89.80(7)$ \\
\hline $\mathrm{O} 7-\mathrm{Na} 1-\mathrm{O} 2^{\mathrm{iv}}$ & $102.26(9)$ & $\mathrm{Na} 2^{\mathrm{vii}}-\mathrm{O} 3-\mathrm{Na} 1^{\text {viii }}$ & $110.53(9)$ \\
\hline $\mathrm{O}^{\mathrm{i}}-\mathrm{Na} 1-\mathrm{O}^{\mathrm{iv}}$ & $79.94(8)$ & $\mathrm{As} 1-\mathrm{O} 4-\mathrm{Na} 2^{\mathrm{i}}$ & $128.10(12)$ \\
\hline $\mathrm{O} 5^{\mathrm{ii}}-\mathrm{Na} 1-\mathrm{O}^{\mathrm{iv}}$ & $118.47(8)$ & $\mathrm{As} 1-\mathrm{O} 4-\mathrm{H} 2$ & $105(3)$ \\
\hline 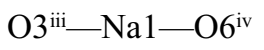 & $83.21(8)$ & $\mathrm{Na} 2 \mathrm{i}-\mathrm{O} 4-\mathrm{H} 2$ & $99(3)$ \\
\hline $\mathrm{O} 7-\mathrm{Na} 1-\mathrm{O}^{\mathrm{iv}}$ & $160.71(8)$ & As $2-\mathrm{O} 5-\mathrm{Na} 1^{\mathrm{vi}}$ & $134.83(12)$ \\
\hline $\mathrm{O} 2^{\mathrm{iv}}-\mathrm{Na} 1-\mathrm{O}^{\mathrm{iv}}$ & $93.76(8)$ & As $2-\mathrm{O} 5-\mathrm{H} 3$ & $111(3)$ \\
\hline $\mathrm{O} 8-\mathrm{Na} 2-\mathrm{O}^{\mathrm{iv}}$ & $156.69(9)$ & $\mathrm{Na} 1^{\mathrm{vi}-\mathrm{O} 5-\mathrm{H} 3}$ & $113(3)$ \\
\hline $\mathrm{O} 8-\mathrm{Na} 2-\mathrm{O} 1^{\mathrm{ii}}$ & $86.89(8)$ & As $2-\mathrm{O} 6-\mathrm{Na} 1^{\mathrm{vii}}$ & $122.07(11)$ \\
\hline $\mathrm{O}^{\mathrm{iv}}-\mathrm{Na} 2-\mathrm{O} 1^{\mathrm{ii}}$ & $90.22(8)$ & $\mathrm{As} 2-\mathrm{O} 6-\mathrm{Na} 2^{\mathrm{i}}$ & $128.58(11)$ \\
\hline $\mathrm{O} 8-\mathrm{Na} 2-\mathrm{O}^{\mathrm{i}}$ & $122.07(8)$ & $\mathrm{Na} 1^{\mathrm{vii}}-\mathrm{O} 6-\mathrm{Na} 2^{\mathrm{i}}$ & $90.36(7)$ \\
\hline $\mathrm{O}^{\mathrm{iv}}-\mathrm{Na} 2-\mathrm{O} 6^{\mathrm{i}}$ & $77.53(7)$ & As $2-\mathrm{O} 7-\mathrm{Na} 1$ & $137.29(12)$ \\
\hline 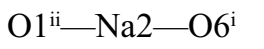 & $126.96(8)$ & As $2-\mathrm{O} 7-\mathrm{Na} 2^{\mathrm{ix}}$ & $126.26(11)$ \\
\hline $\mathrm{O} 8-\mathrm{Na} 2-\mathrm{O} 2^{\mathrm{iv}}$ & $92.76(8)$ & $\mathrm{Na} 1-\mathrm{O} 7-\mathrm{Na} 2^{\mathrm{ix}}$ & $90.85(7)$ \\
\hline $\mathrm{O}^{\mathrm{iv}}-\mathrm{Na} 2-\mathrm{O} 2^{\mathrm{iv}}$ & $63.95(7)$ & As2-O $7-\mathrm{H} 4$ & $114(3)$ \\
\hline $\mathrm{O} 1^{\mathrm{ii}}-\mathrm{Na} 2-\mathrm{O} 2^{\mathrm{iv}}$ & $81.03(8)$ & $\mathrm{Na} 1-\mathrm{O} 7-\mathrm{H} 4$ & $102(3)$ \\
\hline $\mathrm{O} 6^{\mathrm{i}}-\mathrm{Na} 2-\mathrm{O} 2^{\mathrm{iv}}$ & $132.91(8)$ & $\mathrm{Na} 2^{\mathrm{ix}}-\mathrm{O} 7-\mathrm{H} 4$ & $61(3)$ \\
\hline
\end{tabular}


supporting information

$\mathrm{O} 8-\mathrm{Na} 2-\mathrm{O} 4^{\mathrm{i}}$

$\mathrm{O} 3^{\mathrm{iv}}-\mathrm{Na} 2-\mathrm{O} 4^{\mathrm{i}}$
$73.31(7)$ $104.07(8)$

$137.60(11)$

As2-O8- $\mathrm{Na} 2$

Symmetry codes: (i) $-x+1,-y+1,-z+1$; (ii) $x,-y+1 / 2, z-1 / 2$; (iii) $x+1, y, z-1$; (iv) $x, y, z-1$; (v) $x-1, y, z$; (vi) $x,-y+1 / 2, z+1 / 2$; (vii) $x, y, z+1$; (viii) $x-1, y$, $z+1$; (ix) $x+1, y, z$.

Hydrogen-bond geometry $\left(\AA,{ }^{\circ}\right)$

\begin{tabular}{lllll}
\hline$D-\mathrm{H} \cdots A$ & $D-\mathrm{H}$ & $\mathrm{H} \cdots A$ & $D \cdots A$ & $D-\mathrm{H} \cdots A$ \\
\hline $\mathrm{O} 2-\mathrm{H} 1 \cdots \mathrm{O}^{\text {vi }}$ & $0.85(2)$ & $1.75(2)$ & $2.595(3)$ & $175(5)$ \\
$\mathrm{O} 4-\mathrm{H} 2 \cdots \mathrm{O} 8$ & $0.83(2)$ & $1.82(2)$ & $2.643(3)$ & $178(4)$ \\
$\mathrm{O} 5-\mathrm{H} 3 \cdots{ }^{\text {ix }}$ & $0.85(2)$ & $1.73(2)$ & $2.566(3)$ & $171(5)$ \\
$\mathrm{O} 7-\mathrm{H} 4 \cdots \mathrm{O}^{\mathrm{x}}$ & $0.85(2)$ & $1.66(2)$ & $2.500(3)$ & $169(4)$
\end{tabular}

Symmetry codes: (vi) $x,-y+1 / 2, z+1 / 2$; (ix) $x+1, y, z$; (x) $-x+2,-y+1,-z+1$. 